\title{
Prevalencia del trastorno succión-deglución en neonatos pretérmino nacidos en el Hospital Universitario de Santander durante 2016
}

\author{
Andrea Patricia Vargas Sanabria* \\ Oscar Leonardo Torra-Barajas** \\ Nathalia Jácome-Pérez*** \\ Claudia Juliana Ramirez Sanchez ${ }^{* * * *}$
}

*Médica. Especialista en Epidemiología. Hospital Internacional de Colombia. Santander. Universidad Autónoma de Bucaramanga. Bucaramanga. Santander. Colombia

**Médico. Clínica gestionar y bienestar. Bucaramanga. Santander. Colombia

***Estudiante X semestre de medicina. Escuela de medicina. Facultad de salud. Universidad Industrial de Santander. Bucaramanga. Santander. Colombia.

****Fonoaudióloga. Especialista en audiología. II Nivel de Especialidad en Epidemiología. Universidad Autónoma de Bucaramanga. Bucaramanga. Santander. Colombia

Correspondencia: Dra. Andrea Patricia Vargas Sanabria. Dirección: Carrera 8a Número 61-132 Parque San Remo 1 casa 4 Bucaramanga. Santander. Colombia Correo electrónico: Andreavargas@fcv.org Teléfono: 3203948043

\section{RESUMEN}

Introducción: la alimentación por vía oral después del nacimiento es llevada a cabo gracias a la adecuada coordinación de la triada funcional succión-deglución-respiración, sin embargo, cuando hay una disfunción en alguna de las variables, se presentan los trastornos de succión-deglución. Existen diversos factores que condicionan a la presencia de estos trastornos, uno de ellos es la prematurez la cual retrasa la adquisición de la coordinación y el desarrollo de cada función de la triada. Objetivo: determinar la prevalencia de trastorno de succión-deglución en neonatos pretérmino nacidos en el Hospital Universitario de Santander de acuerdo al grado de prematurez así como las variables asociadas. Materiales y métodos: estudio observacional de corte transversal. Se realizó una revisión de historias clínicas de pacientes prematuros de la unidad de neonatología del Hospital Universitario de Santander durante el 2016, incluyendo un total de 115 pacientes. Resultados: los pacientes con diagnóstico de trastorno de deglución presentaron medias de edad gestacional, peso y talla mucho menores con respecto al grupo de pacientes que no presentaron este trastorno, al igual que estancias hospitalarias mucho más prolongadas. Variables como trastorno de hipertensión asociada al embarazo, infección materna al momento del parto, abrupcio de placenta, parto por cesárea, síndrome de dificultad respiratoria, sepsis e ictericia neonatal, íleo y anemia se encontraron en mayor porcentaje en pacientes con trastorno de deglución. Conclusiones: existen variables que se encuentran asociadas con el trastorno de succión-deglución, las cuales deben ser identificadas e intervenidas para mitigar la carga de la patología. MÉD.UIS. 2017;30(3):73-81.

Palabras clave: Trastornos de Deglución. Recién Nacido Prematuro. Edad Gestacional.

\section{Prevalence of suction-swallowing disorders of preterm newborn infants in Hospital Universitario de Santander and its associated variables during 2016}

Introduction: the oral feeding after birth is carried out by proper coordination of the functional suction-swallow-breathing triad; however, when there is a dysfunction in any of the variables, the suction-swallowing disorders are presented. There are several factors which predetermines the presence of these disorders, one of them is prematurity which delays the acquisition of coordination and development of each function of the triad. Objective: to determine the prevalence of suction-swallowing disorder in preterm infants born at the University Hospital of Santander according to the degree of prematurity as well as the associated variables. Materials and methods: observational cross-sectional study. A clinical history review of preterm neonates at the University Hospital of Santander during 2016, including a total of 115 patients, was carried out. Results: patients with a diagnosis of swallowing disorder had significantly lower mean gestational age, weight and height compared to the group of patients who did not present this disorder, as well as longer hospital stays. Variables such as hypertension associated with pregnancy, maternal infection at the time of delivery, abruptio of placenta, cesarean delivery, respiratory distress syndrome, sepsis and neonatal jaundice, ileus and anemia were found to be higher in patients with swallowing disorder. Conclusions: there are variables that are associated with suction-swallowing disorder, which must be identified and intervened to mitigate the burden of the disease. MÉD.UIS. 2017;30(3):73-81.

Keywords: Deglutition Disorders. Infant, Premature. Gestational Age. 
¿Cómo citar este artículo?: Vargas A, Torra-Barajas O, Jácome-Pérez N, Ramírez C. Prevalencia del trastorno succión-deglución en neonatos pretérmino nacidos en el Hospital Universitario de Santander durante 2016. MÉD.UIS. 2017;30(3):73-81.

\section{INTRODUCCIÓN}

Se define como prematuro a todo nacimiento que se produce antes de las 37 semanas de gestación cumplidas; la OMS divide a los prematuros en cuatro grandes grupos de acuerdo a su edad gestacional. El primero, prematuro general que se define como el nacimiento del producto de la gestación menor a 37 semanas, seguido por el prematuro tardío que es aquel que nace dentro de la semana 34 a la 36 con seis días, el tercero, muy prematuro son los nacidos antes de las 32 semanas de gestación y el extremadamente prematuro que corresponde al nacimiento antes de las 28 semanas de gestación'.

La incidencia de nacimientos prematuros ha venido en incremento progresivo no solo por el aumento de la población global sino también por factores asociados al embarazo que predisponen a que la culminación del mismo sea prematura, según las cifras de la OMS cada año nacen aproximadamente 15 millones de niños prematuros y se estima una tasa de nacimientos prematuros que oscila entre el $5 \%$ y el $18 \%$ de los recién nacidos ${ }^{1,2}$. Para el 2009 se reportó que el $11,4 \%^{3}$ de los recién nacidos en Colombia correspondieron a nacimientos prematuros, cifra que aumentó al 20,3\% para el $2016^{4}$.

La supervivencia de los pacientes prematuros a su vez se ha incrementado gracias a avances científicos en el abordaje de problemáticas periparto como lo son la maduración pulmonar in útero con el uso de esteroides, avances en reanimación neonatal y el uso de técnicas que permitan una mejor adaptación neonatal a pesar de las morbilidades neonatales como lo son el uso de surfactante, manejo de soporte ventilatorio, entre otros; este incremento de supervivencia ha impactado predominantemente en el grupo de recién nacidos muy prematuros, en los que se han alcanzado incluso tasas de supervivencia del $90 \%$ si el peso del neonato es igual o superior a $1000 \mathrm{~g}^{5,6}$. Todo esto conlleva a que presenten mayores problemas en el área de la neonatología relacionada con el número elevado de partos prematuros que son un reto para el personal de salud como lo son las morbilidades que acarrea en sí la prematurez; entre los estados mórbidos más comunes encontrados en los prematuros está el Trastorno de SucciónDeglución (TSD)?.

La deglución se entiende como la actividad encaminada a transportar alimento sólido, líquido y saliva desde la boca hasta el estómago, este proceso es llevado a cabo gracias a las fuerzas y los movimientos sincrónicos de los músculos y componentes orofaringolaríngeos ${ }^{2,7}$. La nutrición oral del prematuro no es posible hasta la adquisición de los reflejos orales siendo los más prioritarios succión - deglución, búsqueda, tusígeno y nauseoso, si alguno de estos no se encuentra presente o su respuesta es muy lábil genera que se presente en el Recién Nacido Pretérmino (RNPT) trastornos de la deglución o incoordinación de la triada funcional (succión-deglución-respiración), afectando la fisiología del sistema estomatognático, debido al retraso de la maduración funcional ${ }^{8}$.

La edad gestacional corregida mínima en la que los RNPT pueden tener una alimentación oral exitosa es incierta9. Al no existir criterios establecidos para definir el inicio de la alimentación oral, existen diversas y diferentes recomendaciones con respecto a la edad gestacional más apropiada para inciar la vía oral. Aunque en general los RNPT requieren menos tiempo para lograr una transición completa a una alimentación oral segura contrario a los neonatos de menos edad gestacional, esta no es una regla, dado que la evolución depende de múltiples factores propios del neonato como dependientes de la madre y la práctica clínica, dentro de estos se describen los estados mórbidos asociados con el neonato, el estímulo neonatal anteparto y posparto, la adecuada técnica de lactancia, el compromiso y la disponibilidad por parte del núcleo familiar y del personal de salud para la realización de terapia continua, entre otros ${ }^{10}$.

Hayquerecalcarquees desumaimportanciaidentificar a los recién nacidos pretérmino con TSD debido a que esta deficiencia aumenta las comorbilidades como: la desnutrición, riesgo de bronco-aspiración, neumonías y estancias hospitalarias prolongadas que a su vez predisponen a la presencia de estados mórbidos que empeoran la calidad de vida tanto del paciente como del núcleo familiar e incrementan 
el riesgo de mortalidad y los gastos en salud ${ }^{8,11-13}$. El objetivo de este estudio es determinar la prevalencia del TSD de acuerdo al grado de prematurez así como variables que se asocian con este diagnóstico en el Hospital Universitario de Santander.

\section{Materiales y Métodos}

Estudio observacional de corte transversal, realizado a partir de revisión de historias clínicas de pacientes prematuros de la unidad de neonatología del Hospital Universitario de Santander en la ciudad de Bucaramanga desde el primero de enero de 2016 al 31 de marzo de 2016.

El cálculo del tamaño de la muestra para el estudio se realizó tenidendo en cuenta la tasa de natalidad en Santander proporcionada por datos del DANE $2016^{14}$ y la prevalencia de trastorno de deglución en partos prematuros de $12 \%{ }^{15}$, con un valor de confianza de 95\%, asumiendo un error tipo I máximo del 0,05 y un poder estadistico de $90 \%$, obtenidendo un tamaño de muestra mínimo de 114 pacientes.

Como criterios de inclusión se tuvieron en cuenta pacientes con edad gestacional menor a 37 semanas al momento de nacer y como criterios de exclusión se tomaron malformaciones a nivel de cabeza y cuello, malformaciones del tracto digestivo como atresias, síndromes genéticos, hemorragias intracraneales, inestabilidad cardiovascular que impida la vía oral. Se obtuvo información de las historias clínicas que cumplían el criterio de inclusión y no presentaban ningún criterio de exclusión, las variables se pueden observar en la tabla 1.

Se realizó inicialmente una prueba piloto con el 10\% de la muestra para el entrenamiento del correcto llenado del instrumento de recolección, cada historia clínica fue registrada por dos investigadores para después verificar la concordancia de las variables, si había discrepancia en alguna de ellas, un tercer evaluador realizaba la respectiva confirmación de los datos. Las variables fueron tabuladas en una base de datos en el programa Excel, posteriormente para hacer el análisis se transfirió dicha base de datos al programa STATA 12.0 por medio de STATA TRANSFER y a partir de este programa se elaboró un análisis descriptivo de variables cualitativas por medio de porcentajes y de variables cuantitativas empleando medidas de tendencia central; se generó una relación estadística por medio de un análisis bivariado con el uso de la razón de prevalencia y el Chi2 para probar hipótesis de asociaciones de diversas variables con el desenlace que es la presencia de TSD.

Tabla 1. Variables asociadas a trastorno de succión-deglución

\begin{tabular}{|c|c|}
\hline Grado de prematurez & $\begin{array}{l}\text { Prematuro extremo }(<28 \\
\text { semanas) } \\
\text { Muy prematuro ( }>28-<32 \\
\text { semanas) } \\
\text { Prematuro moderado ( }>32-< \\
37 \text { semanas) }\end{array}$ \\
\hline $\begin{array}{c}\text { Variables } \\
\text { sociodemográficas }\end{array}$ & $\begin{array}{l}\text { Sexo del paciente } \\
\text { Régimen de salud del paciente } \\
\text { Edad y escolaridad materna y } \\
\text { paterna }\end{array}$ \\
\hline $\begin{array}{c}\text { Datos } \\
\text { antropométricos }\end{array}$ & Peso y talla al nacimiento \\
\hline Condiciones periparto & $\begin{array}{l}\text { Número de controles } \\
\text { prenatales } \\
\text { Embarazo deseado o planeado } \\
\text { Comorbilidades periparto: } \\
\text { THAE, RPM, TPP, Embarazo } \\
\text { gemelar, infección materna, } \\
\text { abruptio de placenta. }\end{array}$ \\
\hline Vía del parto & Vaginal o cesárea \\
\hline Estancia hospitalaria & En días \\
\hline $\begin{array}{c}\text { Comorbilidades del } \\
\text { prematuro }\end{array}$ & $\begin{array}{l}\text { SDRA, ERGE, Infecciones ( } \\
\text { conjuntivitis, onfalitis, IVU, } \\
\text { neumonía, enterocolitis } \\
\text { necrotizante), sepsis neonatal, } \\
\text { presencia de DAP, íleo, anemia, } \\
\text { ictericia. }\end{array}$ \\
\hline $\begin{array}{l}\text { Diagnóstico de } \\
\text { trastorno de deglución }\end{array}$ & $\begin{array}{l}\text { Por el servicio de } \\
\text { fonoaudiología }\end{array}$ \\
\hline $\begin{array}{l}\text { Necesidad de } \\
\text { hospitalización } \\
\text { prolongada por } \\
\text { presencia de trastorno } \\
\text { de deglución }\end{array}$ & $\begin{array}{l}\text { Hospitalizaciones prolongadas } \\
\text { exclusivas por esta causa. }\end{array}$ \\
\hline
\end{tabular}

THAE: Trastorno hipertensivo asociado al embarazo. RPM: Ruptura prematura de membranas.

TPP: Trabajo de Parto Pretérmino. SDRA: Síndrome de Dificultad Respiratoria.

ERGE: Enfermedad por Reflujo Gastroesofágico. IVU: Infección de Vías Urinarias.

DAP: Ductus Arterioso Persistente.

Fuente: Autores. 


\section{Resultados}

Se analizaron 115 pacientes prematuros de la unidad de neonatología del Hospital universitario de Santander en la ciudad de Bucaramanga, de los cuales el $65,22 \%$ (IC 95\% 0,56-0,74) presentaron trastorno de succión-deglución. El 63,4\% (IC 95\% 0,54.0,72) de los pacientes eran prematuros tardíos, un 31,3\% ( IC 95\% $0,22.0,39$ ) muy prematuros y tan solo un $5,2 \%$ (IC $95 \%$ $0,01-0,93$ ) fueron extremadamente prematuros

De los pacientes que no presentaban TSD, el $87,5 \%$ (IC95\% 0,76-0,98) pertenecían al grupo de prematuros tardíos, el porcentaje restante eran muy prematuros. El 50,6\% (IC 95\% 0,39-0,62) de los pacientes que presentaron TSD pertenecían al grupo de prematuros tardíos, el 41,3\% (IC 95\% 0,290,52 ) pertenecía al grupo de muy prematuros y el porcentaje restante al grupo de extremadamente prematuros, adicionalmente todos los pacientes del grupo de extremadamente prematuros cursaron con TSD. En cuanto al porcentaje de embarazos no deseados en los pacientes con diagnóstico de TSD se presentó un porcentaje mayor con respecto a los pacientes sin este diagnóstico: $62,1 \%$ versus $45,4 \%$.

El sexo masculino predominó en los dos grupos así como la escolaridad por parte de los padres de secundaria o más; la mayoría de las madres y los padres de estos pacientes no eran adolescentes y un gran porcentaje de ambos grupos pertenecía al régimen subsidiado (Ver Tabla 2). La tabla número tres resume los hallazgos en cuanto porcentaje de las variables sociodemográficas tanto del grupo de prematuros que presentaron trastorno de deglución como los del grupo que no lo presentaron con sus respectivos intervalos de confianza.

Tabla 2. Porcentaje de variables sociodemográficas de cada grupo

\begin{tabular}{|c|c|c|c|c|}
\hline Variables & \multicolumn{2}{|c|}{ Pacientes sin trastorno de deglución } & \multicolumn{2}{|c|}{ Pacientes con trastorno de deglución } \\
\hline Sexo & $\begin{array}{l}\text { Masculino 58,6\% } \\
\text { (IC 95\% 0,47-0,70) }\end{array}$ & $\begin{array}{l}\text { Femenino 41,4\% } \\
\text { (IC 95\% 0,29- 0,52) }\end{array}$ & $\begin{array}{l}\text { Masculino 52,5\% } \\
\text { (IC 95\% 0,36-0,08) }\end{array}$ & $\begin{array}{l}\text { Femenino 47,5\% } \\
\text { (IC 95\% 0,31- 0,63) }\end{array}$ \\
\hline $\begin{array}{l}\text { Escolaridad } \\
\text { materna }\end{array}$ & $\begin{array}{l}\text { Primaria } 17,3 \% \\
\text { (IC95\% 0,06-0,34) }\end{array}$ & $\begin{array}{l}\text { Secundaria o más } \\
82,7 \% \text { (IC95\% 0,65- } \\
0,99)\end{array}$ & $\begin{array}{l}\text { Primaria 22,92\% } \\
\text { (IC95\% 0,10-0,35) }\end{array}$ & $\begin{array}{l}\text { Secundaria o más } \\
77,08 \%(I C 95 \% \\
0,64-0,89)\end{array}$ \\
\hline $\begin{array}{l}\text { Grupo etario de } \\
\text { la madre }\end{array}$ & $\begin{array}{l}\text { Adolescente 47,3\% } \\
\text { (IC95\% 0,30-0,64) }\end{array}$ & $\begin{array}{l}\text { No adolescente } \\
52,7 \% \text { (IC95\% 0,35- } \\
0,69)\end{array}$ & $\begin{array}{l}\text { Adolescente } 10 \% \\
(\text { IC95\% } \quad 0,002-0,19)\end{array}$ & $\begin{array}{l}\text { No adolescente } \\
90 \% \text { (IC95\% 0,80- } \\
0,99)\end{array}$ \\
\hline $\begin{array}{c}\text { Escolaridad } \\
\text { paterna }\end{array}$ & $\begin{array}{l}\text { Primaria } 14,2 \% \\
\text { (IC95\% 0,20-0,30) }\end{array}$ & $\begin{array}{l}\text { Secundaria o más } \\
85,8 \% \text { (IC95\% 0,69- } \\
1,02)\end{array}$ & $\begin{array}{l}\text { Primaria } 35 \% \\
(\text { IC95\% } 0,19-0,50)\end{array}$ & $\begin{array}{l}\text { Secundaria o más } \\
65 \% \text { (IC95\% 0,49- } \\
0,80)\end{array}$ \\
\hline $\begin{array}{c}\text { Grupo etario del } \\
\text { padre }\end{array}$ & $\begin{array}{l}\text { Adolescente } 9,5 \% \\
(\text { IC95\% 0,30-0,64) }\end{array}$ & $\begin{array}{l}\text { No adolescente } \\
90,5 \%(\text { IC95\% 0,76- } \\
1,04)\end{array}$ & $\begin{array}{l}\text { Adolescente } 10 \% \\
(\text { IC95\% 0,002-0,19) }\end{array}$ & $\begin{array}{l}\text { No adolescente } \\
90 \% \text { (IC95\% 0,80- } \\
0,99)\end{array}$ \\
\hline $\begin{array}{l}\text { Régimen de } \\
\text { salud }\end{array}$ & $\begin{array}{l}\text { Subsidiado 85\% } \\
\text { (IC95\% 0,73- 0,96) }\end{array}$ & $\begin{array}{l}\text { No subsidiado 15\% } \\
\text { (IC95\% 0,34- 0,36) }\end{array}$ & $\begin{array}{l}\text { Subsidiado 88\% } \\
\text { (IC95\% 0,80- 0,95) }\end{array}$ & $\begin{array}{l}\text { No subsidiado } 12 \% \\
\text { (IC95\% 0,44- 0,49) }\end{array}$ \\
\hline
\end{tabular}

Fuente: Autores. 
Con respecto a las variables cuantitativas, se puede evidenciar que el grupo de pacientes con TSD presentaron medias de edad gestacional, peso y talla mucho menores con respecto al grupo de pacientes sin este trastorno, a su vez se evidenció una media de controles prenatales realizados hasta el momento del nacimiento menor y medias de estancias hospitalarias mucho más prolongadas comparados con las de los pacientes que no presentaron trastorno de deglución (Ver Tabla 3). En las gráficas 1 a la 5 se puede observar los respectivos valores con sus desviaciones estándar.

Tabla 3. Distribución de variables cuantitativas de acuerdo a cada grupo

\begin{tabular}{|c|c|c|}
\hline Variables & Pacientes sin trastorno de deglución & Pacientes con trastorno de deglución \\
\hline $\begin{array}{c}\text { Mediana y rango intercuartíico de } \\
\text { edad gestacional }\end{array}$ & $\begin{array}{c}34,45 \\
\text { semanas } \\
\text { rango intercuartílico: } 2,12\end{array}$ & $\begin{array}{c}31,6 \\
\text { semanas }\end{array}$ \\
\hline rango intercuartílico: 4
\end{tabular}

Fuente: Autores.

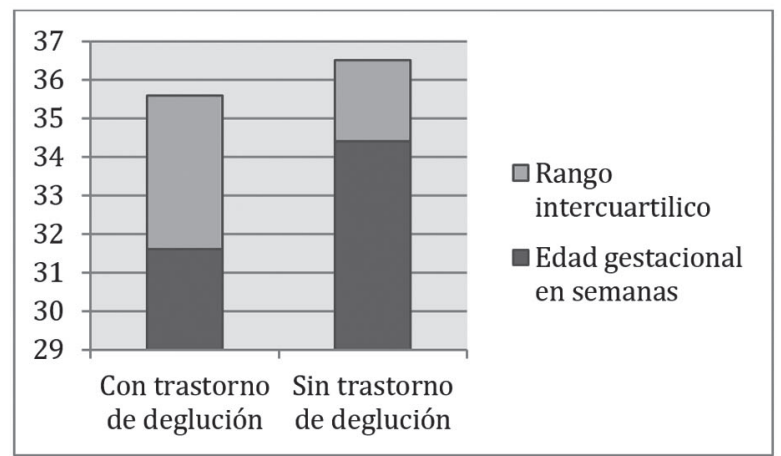

Gráfica 1: Mediana de edad gestacional en semanas con sus rangos intercuartilicos

Fuente: Autores.

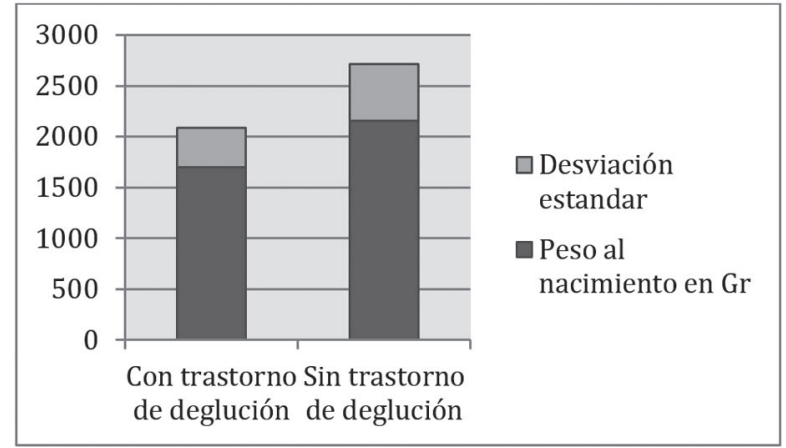

Gráfica 2: Media del peso al nacimiento en gramos con su desviación estandar

Fuente: Autores.

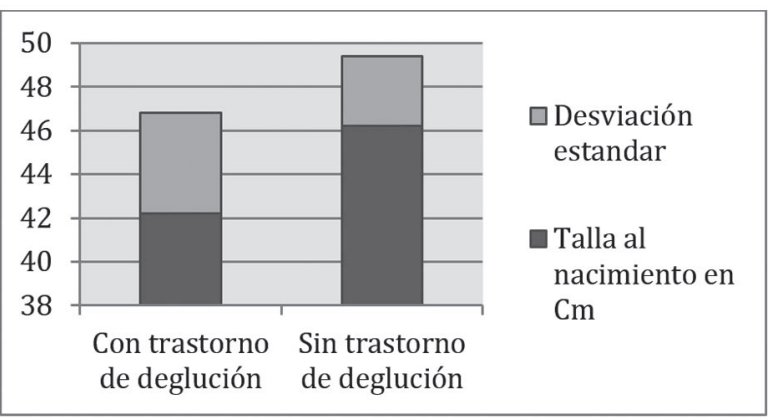

Gráfica 3: Media de la talla al nacimiento en centimetros con su desviación estandar

Fuente: Autores.

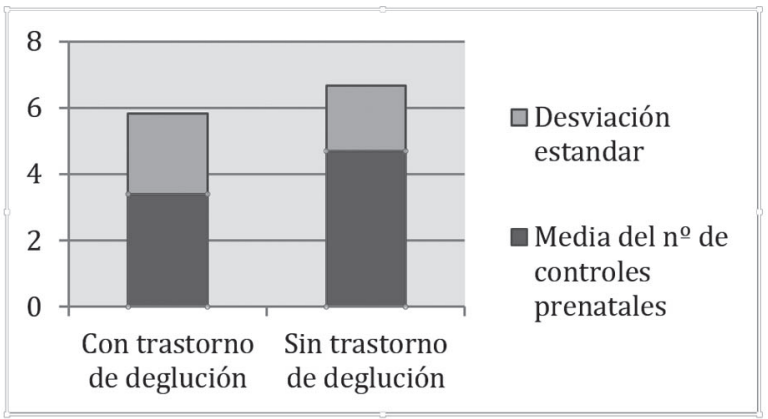

Gráfica 4: Media del número de controles prenatales al nacimiento con su desviación estandar

Fuente: Autores. 


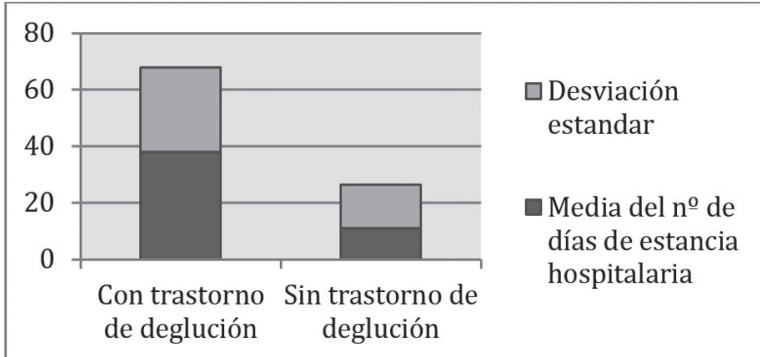

Gráfica 5: Media del número de días de estancia hospitalaria con su desviación estandar

Fuente: Autores.

De las variables cuantitativas, las que en mayor porcentaje se encontraron en pacientes con TSD con respecto a los que no tienen este diagnóstico fueron, trastorno de Hipertensión Asociado al Embarazo (THAE) con un $28 \%$ versus $17,5 \%$, infección materna al momento del parto con un $29,3 \%$ versus $12,5 \%$ abruptio de placentae con un $4 \%$ versus $0 \%$, parto por cesárea con un $69,3 \%$ versus $52,5 \%$, Síndrome de Dificultad Respiratoria Aguda (SDRA) con un 93,3\% versus $67,5 \%$, sepsis neonatal con un $47,3 \%$ versus $22,5 \%$, ictericia neonatal con un $71,6 \%$ versus $52,5 \%$, íleo con un $21,6 \%$ versus $12,5 \%$ y anemia con un $37,8 \%$ versus $5 \%$ (Ver Tabla 4).

Posterior al análisis bivariado por medio de $\mathrm{CHI}$ se encontró las variables que se asocian al trastorno de deglución de manera significativa con sus respectivas $p$, el resto de las variables que no se muestran en la tabla 5 no presentaron una $p$ significativa.

\section{Discusión}

El presente artículo muestra la asociación de variables clínicas y sociodemográficas con la presencia de TSD en pacientes prematuros del servicio de neonatología del Hospital Universitario de Santander, además de que permite evidenciar la carga que presentan los pacientes con este diagnóstico en cuanto a las estancias hospitalarias prolongadas hasta asegurar una vía alimenticia segura, lo cual aumenta directamente los costos hospitalarios, predispone a otros estados mórbidos y disminuye la calidad de vida del núcleo familiar del paciente.

En este estudio se encontró que el TSD tenia una mayor presentación en neonatos pretermino tardíos, lo anterior se relaciona con hallazgos encontrados por Adams-Chapman y colaboradores ${ }^{16}$ en donde documentaron que de los 1477 pacientes prematuros estudiados el $87 \%$ presentaban alguna disfunción
Tabla 4. Distribución de variables cualitativas de acuerdo a cada grupo

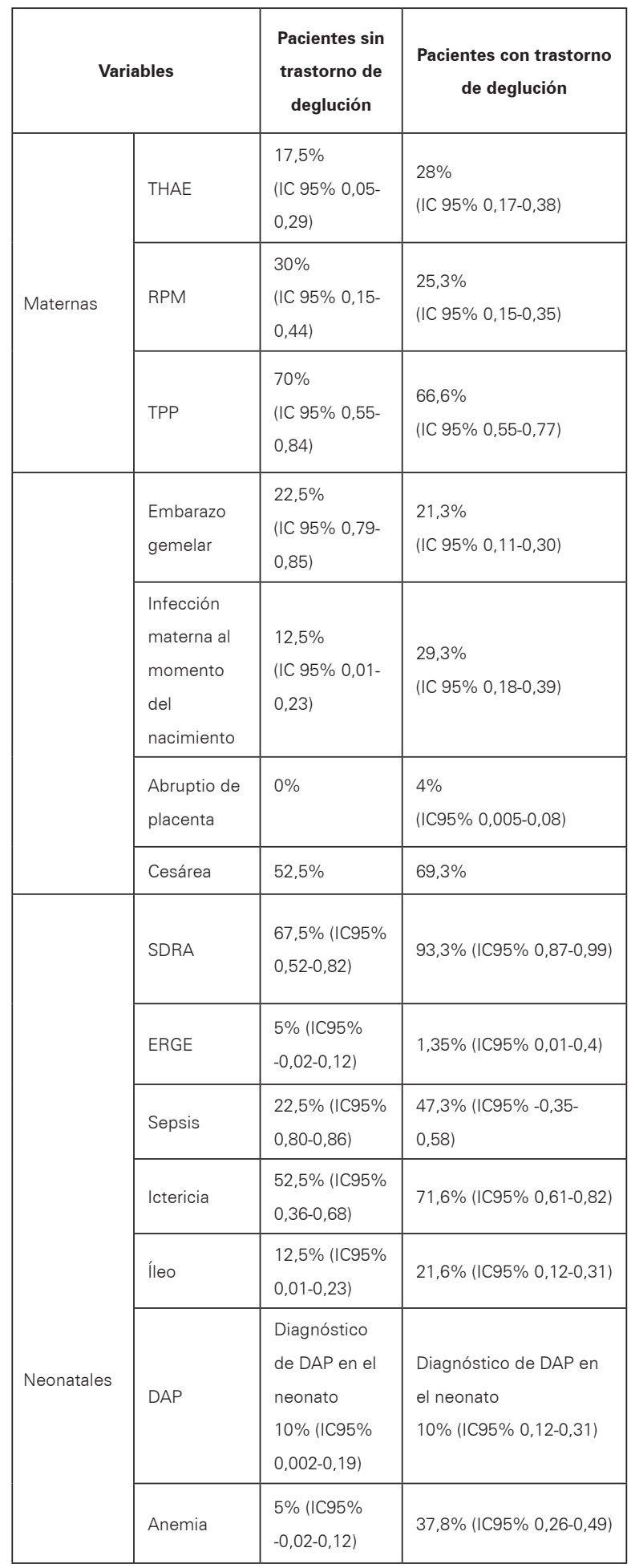

THAE: Trastorno de Hipertensión Asociado al Embarazo. RPM: Ruptura Prematura de Membranas. TPP: Trabajo de Parto Prolongado. SDRA: Síndrome de Dificultad Respiratoria Aguda. ERGE: Enfermedad de Reflujo Gastroesofágico. DAP: Ductus Arterioso Persistente.

Fuente: Autores. 
en su alimentación y que aquellos que presentaban patrones de alimentación más disfuncionales correspondían a los de menor edad gestacional ${ }^{14}$. A diferencia de los pacientes muy prematuros y prematuros tardíos, la totalidad de los pacientes estudiados en este estudio, clasificados como extremadamente prematuros presentaron TSD, lo que permite observar que la edad gestacional se relaciona de manera directa con la adquisición de reflejos y la coordinación de la triada funcional (succión- deglución- respiración) y por lo tanto se asocia de manera inversa con la presencia de TSD como se ha descrito a lo largo de la literatura como en los estudios realizados por Dodrill y Crapnell y colaboradores ${ }^{17}$.

Tabla 5. Distribución de variables estadísticamente significativas asociadas a trastorno de succión- deglución

\begin{tabular}{|c|c|}
\hline Variable & Valor de $p$ \\
\hline Grado de prematurez & 0,001 \\
\hline Peso al nacimiento & 0,001 \\
\hline Talla al nacimiento & 0,001 \\
\hline $\begin{array}{l}\text { Número de controles prenatales al } \\
\text { nacimiento }\end{array}$ & 0,003 \\
\hline Días de estancia hospitalaria & 0,001 \\
\hline $\begin{array}{l}\text { Infección materna al momento del } \\
\text { nacimiento }\end{array}$ & 0,043 \\
\hline SDRA en neonato & 0,001 \\
\hline Sepsis neonatal & 0,009 \\
\hline Ictericia neonatal & 0,041 \\
\hline Anemia en neonato & 0,001 \\
\hline
\end{tabular}

Fuente: Autores.

Adicionalmente, en una revisión de la literatura realizada por Pagliario y colaboradores ${ }^{18}$, de los 17 artículos incluidos, 10 analizaron la relación directa entre distintas variables y el desarrollo de TSD. Dentro de estas se describieron el reflujo gastroesofágico, las complicaciones pulmonares, el bajo peso al nacer, la menor edad gestacional, el uso prolongado de oxígeno suplementario, entre otras.
En relación al contexto sociodemográfico Ilama la atención que el 56,5\% (IC 95\% 0,47-0,65) pertenecían al sexo masculino, incluso de los pacientes que presentaron trastorno de deglución el 58,6\% (IC $95 \% 0,47-0,70$ ) eran de sexo masculino, similar a lo encontrado por otros autores como lo fueron Diaz y colaboradores en el estudio realizado en la Habana donde documentaron un 51,79\% de población masculina en su muestra de pacientes con TSD ${ }^{19}$, sin embargo no se es posible concluir que exista alguna relación entre el sexo y la presencia de TSD. No obstante, de acuerdo a las cifras proporcionadas por el Departamento Administrativo Nacional de Estadistica con corte a 31 de marzo de 2017 en Colombia $^{20}$, se registró un mayor número de nacimientos de sexo masculino para el año 2016, lo que explicaría el mayor porcentaje de recién nacidos de este sexo en el estudio.

Contrario a lo encontrado en la literatura, la escolaridad materna que predominó tanto en los prematuros con TSD como en los prematuros que no tenían este diagnóstico fue secundaria o estudios superiores a esta fue de 77,08\% (IC 95\% 0,64-0,89) y $82,6 \%$ (IC 95\% 0,65-0,99) ) respectivamente, al igual que la escolaridad paterna en pacientes prematuros con TSD y prematuros sin este diagnóstico que correspondió a $65 \%$ (IC 95\% 0,49-0,80) y 85,7\% (IC 95\% 0,69-1,02) respectivamente, igualmente sucedió con la edad de los padres, se evidenció que tanto en los pacientes con TSD como en los que no tenían este diagnóstico predominaron las madres no adolescentes ( 63,5\% (IC 95\% 0,52-0,74) y 52,6\% (IC 95\% $0,35-0,69)$ respectivamente) al igual que los padres no adolescentes ( $90 \%$ (IC 95\% 0,80-0,99) y 90,4\% (IC $0,76-1,04)$ respectivamente $\mathrm{e}^{19,21}$.

En este estudio, el régimen de salud se utilizó como indicador de nivel socioeconómico. En general la gran mayoría de pacientes que presentaban TSD y aquellos que no lo presentaron hacían parte de un núcleo familiar con estrato socioeconómico bajo $88 \%$ (IC 95\% 0,80-0,95) y $85 \%$ (IC 95\% 0,73-0,96) respectivamente, esto se relaciona con que los nacimientos prematuros pueden asociarse a las dificultades que presentan las maternas de bajos estratos socioeconómicos con el acceso al sistema de salud y por tanto la presencia de mayor número de morbilidades no tratadas que conllevan a partos prematuros. De acuerdo a Crapnell y colaboradores en el 2013 ${ }^{21}$, en donde también se empleo el régimen de salud como un aproximador del nivel socioeconómico, el 
$61 \%$ de pacientes incluidos pertenecían al régimen de salud subsidiado, encontrando asociación estadísticamente significativa entre el estrato socioeconómico bajo con trastornos de deglución $(p=0,046)$

De acuerdo al análisis multivariado se encontró que la edad gestacional corresponde a un factor importante relacionado con el desarrollo de TSD. A lo largo de la literatura se describe que a menor edad gestacional, medidas antropométricas menores y menor número de controles prenatales hay mayor predisposición al desarrollo de estados mórbidos perinatales, entre ellos factores que conlleven a la presencia de TSD en la etapa neonatal. De acuerdo al estudio realizado por Wrotniak y colaboradores, se documentó que los neonatos extremadamente prematuros de bajo peso presentaron mayor riesgo de problemas en la maduración adecuada para realizar una alimentación nutritiva en comparación con los menos prematuros 22 .

Los resultados del presente artículo no son la excepción a este comportamiento, la media de la edad gestacional al momento del nacimiento de los pacientes con trastorno de deglución fue mucho menor (31,71 semanas DS 2,56 (IC 95\% 31,1$32,3)$ ) comparada con la de los pacientes que no presentaron este trastorno (34,08 semanas DS 1,76 (IC 95\% 33,5-34,6)); tendencia que se observó en otras variables como media del peso al momento del nacimiento de los pacientes que presentaban trastorno de deglución versus neonatos que no presentaban el diagnóstico ( 1703,53 g DS 560,9 (IC $95 \% 1574,4-1832,5$ ) versus 2156 g DS 380, 5 (IC 95\% 2033$2279,8)$ respectivamente); así como en la media de la talla al momento del nacimiento ( 42,2 centímetros DS 4,6 (IC 95\% 41,1- 43,3) versus 46,2 Centímetros DS 3,2 (IC 95\% 45,1- 47,3) respectivamente). A su vez la media del número de controles prenatales realizados hasta el momento del nacimiento de los pacientes que presentaban trastorno de deglución fue menor con respecto a los que no presentaban el diagnóstico ( 3,4 controles DS 2,42 (IC 95\% 2,833,98 ) versus 4,7 controles DS 1,98 (IC 95\% 4,05-5,36) respectivamente). En las variables anteriormente descritas, se encontró una relación estadísticamente significativa con el TSD como se puede evidenciar en la tabla 5 .

da Costa y colaboradores ${ }^{23}$, describen la asociación entre menor peso al nacimiento con el desarrollo de TSD. Los recién nacidos pretérmino, en particular aquellos descritos como pequeños para la edad gestacional presentaban un riesgo aumentado para TSD. Se describen que estos pacientes con bajo peso y que nacen antes de término presentan dos desventajas, la prematuridad y el incremento en las necesidades energéticas para el crecimiento, factores que influyen en la presencia de estas patologías.

da Costa

De acuerdo a los resultados obtenidos los recién nacidos de madres que presentaron infecciones maternas al momento del nacimiento presentaron en mayor proporción TSD y así mismo se encontró que la presencia de SDRA en neonato $(p=0,000)$, sepsis neonatal $(p=0,009)$, ictericia $(p=0,041)$ y anemia $(p=0,001)$ neonatal se asocia con TSD, sin embargo no se cuenta con más estudios en la literatura científica que evalúen la asociación de estas variables con TSD.

Finalmente se documentó que aquellos pacientes con diagnóstico de trastorno de succión deglución presentaban medias de estancia hospitalaria mayores que aquellos que no tenían este diagnóstico (38 días DS 29,8 (IC 95\% 31,1- 44,9) y 11,16 días DS 15,2 (IC 95\% 6,02-16,3) respectivamente), esto se correlaciona con los hallazgos realizados en el estudio de Lau y colaboradores ${ }^{24}$ en el cual se estimó la relación entre la dificultad para alcanzar una adecuada alimentación oral y la duración de la estancia hospitalaria, encontrando que los recién nacidos de 35 semanas, en comparación con los nacidos a las 34 semanas de gestación presentaron mayor dificultad para una adecuada alimentación oral $(p=0,035)$ y mayor estancia hospitalaria $(p<0,001$ ); esto se relaciona con el incremento de días que los pacientes prematuros deben permanecer en la unidad de neonatología hasta que adquieran un adecuado desarrollo de los reflejos, una adecuada coordinación de la triada funcional (succióndeglución- respiración) y por lo tanto se garantice una vía alimenticia segura; sin embargo, las estancias hospitalarias prolongadas pueden predisponer al incremento de riesgo de estados mórbidos adquiridos en el ámbito hospitalario como lo son las infecciones nosocomiales, a su vez incrementa los costos hospitalarios y disminuye la calidad de vida del entorno familiar del paciente, de allí radica la necesidad de identificar las variables que se asocian a esta problemática para realizar una intervención multidisciplinaria oportuna que disminuya la carga de esta patología. La principal limitación de este estudio es que fue realizado en una única institución, de tercer nivel de atención de carácter público que 
presta sus servicios en su mayoría a personas de bajo nivel socioeconómico.

\section{CONCLUSIONES}

Los pacientes con trastorno de succión-deglución presentaron proporciones de peso, talla y clasificación de grado de prematurez menor que los recién nacidos pretérmino que no presentaron el trastorno. Adicionalmente, una alta prevalencia de los pacientes con trastorno de succión-deglución tuvo menor número de controles prenatales, desarrollaron síndrome de dificultad respiratoria aguda secundario a enfermedad de membrana hialina, requirieron hospitalizaciones prolongadas hasta garantizar una vía oral segura y efectiva sin importar que el resto de sus comorbilidades ya hubieran sido resueltas. Además, se encontró asociación estadisticamente significativa para el desarrollo de trastornos de succción-deglución con variables como sepsis, ictericia, anemia en el neonato e infección materna en el momento del parto. Se recomienda tener presente las variables que tuvieron asociación con el trastorno de succión-deglución con el fin de realizar abordajes oportunos y efectivos, así como brindar educación a los padres para que se involucren en la rehabilitación y de esta manera disminuir la ansiedad y mejorar el vínculo familiar.

\section{RefERENCIAS BibLIOGRÁFICAS}

1. World Health Organization [Internet]. Preterm birth. WHO media centre: fact sheets (publicadas en noviembre de 2016) [Citado 15 Jul 2017]. Disponible en: http://www.who.int/mediacentre/ factsheets/fs363/en/

2. The Lancet. The global burden of preterm birth. Lancet [Internet]. 2009 [Citado 15 Jul 2017];374(9697):1214. Available from: http:// dx.doi.org/10.1016/S0140-6736(09)61762-1

3. Palencia A. Parto prematuro. Sociedad colombiana de pediatría. 2009;9(4):10-9.

4. DANE [Internet]. Nacimientos 2016 - Preliminar. Cifras con corte a 31 de marzo de 2017 (publicadas el 30 de junio de 2017). Nacimientos por área de ocurrencia y sexo, según grupos de edad de la madre, total nacional [Citado 15 Jul 2017]. Disponible en: https://www.dane.gov.co/index.php/estadisticas-por-tema/salud/ nacimientos-y-defunciones/nacimientos/nacimientos-2016

5. Merritt TA, Pillers D, Prows SL. Early NICU discharge of very low birth weight infants: A critical review and analysis. Semin Neonatol. 2003;8(2):95-115.

6. O'Shea TM, Klinepeter KL, Goldstein DJ, Jackson BW, Dillard RG. Survival and developmental disability in infants with birth weights of 501 to 800 grams, born between 1979 and 1994 . Pediatrics. 1997;100(6):982-6.

7. Campos-montero ZI. Problemas de la alimentación en lactantes. Segunda parte : fases oral y faríngea. Acta pediatría costarricense. 2010;(22)1:14-22.

8. La Orden Izquierdo E, Salcedo Lobato E, Cuadrado Pérez I, Herráez Sánchez MS, Cabanillas Vilaplana L. Retraso de la adquisición de la succión-deglución-respiración en el pretérmino; efectos de una estimulación precoz. Nutr Hosp. 2012;27(4):1120-6.

9. Fucile S, Gisel E, Lau C. Oral stimulation accelerates the transition from tube to oral feeding in preterm infants. J Pediatr [Internet]. 2002 [Citado 15 Jul 2017]; 141(2):230.

10. Maastrup R, Hansen BBM, Kronborg H, Bojesen SN, Hallum K, Frandsen A, et al. Breastfeeding progression in preterm infants is influenced by factors in infants, mothers and clinical practice: the results of a national cohort study with high. PLoS One [Internet]. 2014 [Citado 15 Jul 2017];9(9):e108208. Available from: http://dx.plos.org/10.1371/journal.pone.0108208

11. Hardy W. Evidence-Based Practice Brief: Promoting breastfeeding in NICU. Adv Neonatal Care [Internet]. 2010 [Citado 15 Jul 2017];10(1):40. Available from: https://www.ncbi.nlm.nih.gov/ pubmed/20150781

12. Johnson AN. Engaging fathers in the NICU: taking down the barriers to the baby. J Perinat Neonatal Nurs [Internet]. 2008 [Citado 15 Jul 2017];22(4):302-6. Available from: http://www. ncbi.nlm.nih.gov/pubmed/19011495

13. Golianu B, Krane E, Seybold J, Almgren C, Anand KJS. Non-Pharmacological Techniques for Pain Management in Neonates. Semin Perinatol [Internet]. 2007 [Citado 15 Jul 2017];31(5):318-22. Available from: http://dx.doi.org/10.1053/j. semperi.2007.07.007

14. Alcaldía de Bucaramanga. Plan de Desarrollo 2016-2019. Planes y proyectos (publicadas en 2016) [Citado 15 Jul 2017]. Disponible en: http://versionantigua.bucaramanga.gov.co/documents/ dependencias/PLAN_COMPLETO_2016-2019.pdf

15. Blencowe $\mathrm{H}$, Cousens $\mathrm{S}$, Oestergaard M, Chou D, Moller AB, Narwal R, et al. National, regional and worldwide estimates of preterm birth rates in the year 2010 with time trends since 1990 for selected countries: a systematic analysis and implications. The Lancet [Internet]. 2012 [Citado 15 Jul 2017]; 379(9832):216272. Disponible en: http://www.thelancet.com/journals/lancet/ article/PIIS0140-6736(12)60820-4/abstract

16. Adams-Chapman I, Bann CM, Vaucher YE, Stoll BJ. Association between feeding difficulties and language delay in preterm infants using bayley scales of infant development-third edition. J Pediatr [Internet]. 2013 [Citado 15 Jul 2017];163(3):680-5. Disponible en: http://dx.doi.org/10.1016/j.jpeds.2013.03.006

17. Dodrill P. Feeding difficulties in preterm infants. ICAN Infant, Child, Adolesc Nutr. 2011;3(6):324-31.

18. Pagliaro CL, Bühler KEB, Ibidi SM, Limongi SCO. Dietary transition difficulties in preterm infants: Critical literature review. J Pediatr (Rio J) [Internet]. 2016 [Citado 15 Jul 2017];92(1):7-14. Disponible en: http://dx.doi.org/10.1016/j.jped.2015.05.004

19. Diaz Y. Repercusión de alteraciones del proceso de la deglución en la comunicación master's thesis. La Habana: Instituto Superior De Ciencias Médicas De La Habana; 2007. 78p Tomado de: http:// www.sld.cu/galerias/pdf/sitios/rehabilitacion-logo/repercusion del_proceso_de_deglucion_en_la_comunicacion.pdf

20. DANE. Nacimientos 2016 - Preliminar. Crifras con corte a 31 de marzo de 2017 (publicadas el 30 de junio de 2017). Nacimiento por tiempo de gestación según departamento, municipio y área de residencia de la madre. Consultado el 15 de julio de 2017. Disponible en: https://www.dane.gov.co/index.php/estadisticaspor-tema/salud/nacimientos-y-defunciones/nacimientos/ nacimientos-2016

21. Crapnell TL, Rogers CE, Neil JJ, Inder TE, Woodward LJ, Pineda RG. Factors associated with feeding difficulties in the very preterm infant. Acta Paediatr. 2013;102(12):e539-45.

22. Wrotniak BH, Stettler N, Medoff-Cooper B. The relationship between birth weight and feeding maturation in preterm infants. Acta Paediatr [Internet]. 2009 [Citado 15 Jul 2017];98(2):28690. Disponible en: https:/www.ncbi.nlm.nih.gov/pmc/articles/ PMC2692498/

23. da Costa SP, van der Schans CP, Zweens MJ, Boelema SR, van der Meij E, Boerman MA, et al. The Development of Sucking Patterns in Preterm, Small-for-Gestational Age Infants. J Pediatr [Internet]. 2010 [Citado 15 Jul 2017];157(4):603-9. Disponible en: http:// linkinghub.elsevier.com/retrieve/pii/S0022347610003379

24. Chantal L. Development of infant oral feeding skills : What do we know ? Development of infant oral feeding skills : what do we know? Am J Clin Nutr. 2016;103:616-21. 